\title{
Ultrasonographic Characteristics of Cervical Nerve Roots in Patients with the Degeneration of Cervical Vertebra
}

\author{
Fang Luo ${ }^{1, \dagger}$, Jun Chen ${ }^{1,2, \dagger}$, Shan $\mathrm{Wu}^{1,{ }^{*}}$, Shan $\mathrm{He}^{1}$ \\ ${ }^{1}$ Department of Neurology, The Affiliated Hosptial of Guizhou Medical University, Guiyang, China \\ ${ }^{2}$ Department of Clinical Medicine, The Third People's Hospital of Yuhang District, Hangzhou, China \\ Email address: \\ wuwushan@163.com (Shan Wu) \\ ${ }^{*}$ Corresponding author \\ $\uparrow$ Fang Luo and Jun Chen are co-first authors.
}

\section{To cite this article:}

Fang Luo, Jun Chen, Shan Wu, Shan He. Ultrasonographic Characteristics of Cervical Nerve Roots in Patients with the Degeneration of Cervical Vertebra. International Journal of Medical Imaging. Vol. 9, No. 3, 2021, pp. 141-148. doi: 10.11648/j.ijmi.20210903.12

Received: June 15, 2021; Accepted: July 5, 2021; Published: July 21, 2021

\begin{abstract}
Background: Brachial plexus injury is a complex nerve injury. This may be due to the anatomical complexity of the brachial plexus. Purpose: To obtain sonographic characteristics and oblique sagittal diameter of cervical nerve root by high frequency ultrasound, and the morphology of brachial plexus was described. Methods: Two hundred and twenty-one subjects were recruited in our ultrasonography laboratory between March and December 2016. Their ultrasonographic characteristics of bilateral brachial plexus and cervical nerve roots were obtained by ultrasound, and they were measured with high echo sites on the bone cortex of anterior or posterior tubercles as positioning points in oblique sagittal. Results: 1. Cross-sectional sonogram of brachial plexus showed round and (or) oval "uniform" hypoechoic masses in the gap between anterior scalene muscle with middle scalene muscle, which were arranged in an arc shape; uniform low echo detected in short axis section and long axis section imaging of nerve bundles, which were wrapped by slightly hyper echoic epineuria. 2. Ultrasonographic imaging demonstrated that cervical nerve roots arranged between anterior tubercle and posterior tubercle, and the displaying rate of nerve roots of $\mathrm{C} 4$ to $\mathrm{C} 7$ was $100 \%$; the displaying rate of nerve root of $\mathrm{C} 8$ was $78 \%$. 3. Normal values of the diameters of cervicalr root nerves at intervertebral foramen were: $\mathrm{C} 4,2.65 \pm 0.27 \mathrm{~mm}$; C5, $3.33 \pm 0.33 \mathrm{~mm}$; C6, $3.76 \pm 0.36 \mathrm{~mm}$; C7, $4.84 \pm 0.30 \mathrm{~mm}$; C8, $3.48 \pm 0.34 \mathrm{~mm}$. Conclusion: 1. Oblique sagittal imaging, nerve root of $\mathrm{C} 7$ is the thickest among all nerve roots of all examinee; 2 . The diameter that of cervical nerve root of $\mathrm{C} 4$ may be positively correlated with the height.
\end{abstract}

Keywords: High Frequency Ultrasound, Cervical Spondylosis, Degeneration of Cervical Vertebra, Cervical Nerve Root, Magnetic Resonance Imaging, Intervertebral Foramen

\section{Introduction}

Reliable assessment of brachial plexus disorders can be challenging due to the complexity of the anatomy and variation of potential pathology [1]. The brachial plexus is a complex network of nerve roots (C5-T1) that coalesce into proximal trunks, then divided into cords and distal branches, from the neck to axilla [2]. Ultrasound technology has expanded substantially over the past few years. As a clinical tool, it has been well used in many different medical areas, which has both diagnostic, therapeutic uses and predict prognosis [3]. MRI often shows multilevel compression; In addition, the degree of nerve swelling distal to the compression in its extra spinal segment as assessed by ultrasonography may better indicate which cervical nerve roots (NRs) is most affected. However, MRI is expensive, and it is static. MRI is also contraindicated in patients with a pacemaker, defibrillator or deep brain stimulator. High-frequency ultrasound transducers have the ability to produce superior, high-resolution images and membrane, allowing for clearly depiction of nerve bundles anatomy [4]. In recent years, nerve plexus ultrasound has become an irreplaceable method for anesthesia - guided nerve block. Cervical radiculopathy is a common condition that usually results from compression and inflammation of the cervical nerve root or roots in the region of the neural foramen. It is 
frequently caused by cervical disc protrusion and cervical spondylosis [5]. Of course, They are complementary inspection for examining the brachial plexus, ultrasound (US) and magnetic resonance imaging (MRI) are complementary investigations. US is well advantages for screening most extraforaminal pathologies, whereas MRI is more sensitive and accurate for specific clinical indications because of its high resolution [6]. Cervical disc degenerative changes or protrusion is commonly observed on magnetic resonance images in healthy subjects. However, large-scale studies have been rare, and the frequency and range of these findings in healthy subjects have not been clarified. Moreover, there are no reports regarding the correlation between cervical disc degeneration, Relationship between cervical nerve root diameter and intervertebral disc herniation. To date, however, this association is relatively few in clinical studies. Recently, sonographic imaging assessment has been considered an new alternative method for evaluating cervical root and trunk lesions. With the advancement of diagnostic ultrasound equipment and technology, and the technique requirements of patient for noninvasive.

Of course, focuing on superficial soft tissue visualized by ultrasound, where has been noticed by clinicians and patients [7]. Ultrasonography is the practice of using sound waves to create an ultrasonic image. Ultrasonic assessment has been considered an alternative simple and noninvasive method for evaluating superfical organ, such as peripheral nerve, cervical root lesions, trunk lesions and so on. The higher the frequency of the transducer, the better the image resolution. A high-frequency $(12+\mathrm{MHz})$ linear array transducer is primarily used in the evaluation of neuromuscular structures, particularly nerves. Although existing studies have shown that high-frequency ultrasound can be used successfully for brachial plexus block, little has beendone to describe the contorts and diameter of the brachial plexus. In particular, there is no study on the brachial plexus root of normal diameter out of the intervertebral foramen [8]. A major advance in nerve anatomy description has been made with the development of high-resolution ultrasound-imaging techniques [2]. Ultrasound technology has expanded substantially over the past few years. As a clinical tool, it has been well used in many different medical fields, which has both diagnostic and therapeutic uses [3]. Ultrasound may be an important supplement to electro diagnostics in evaluating brachial plexopathies [9]. Ultrasoung is a valuable imaging technique in patients with suspected or known to have brachial plexus nerve damage. It help to identify the damaging degree, predict prognosis and responsiveness to therapy and affect management as well follow-up and most recently as primary prevention diagnostic tool. Further researches should focus on examination of brachial plexus nerve roots thus providing more diagnostic value with more benefit to the clinicians for management of their patients.

\section{Materials and Methods}

The institutional review committee approved the study and has obtained the informed consent of all participants. All subjectss underwent brachial plexus high-frequency ultrasound. Inclusion criteria were: (1) in nearly 3 months in our hospital for cervical MRI (who showed degeneration of cervical vertebra, diagnosed as cervical spondylarthrosis patients, including the Patients from the outpatient department of Neurology and inpatients from March 2016 to December 2016 in the Guizhou Medical University affiliated hospital). (2) all the subjects had no clinical signs or symptoms of cervical radiculopathy, such as arm, scapular or periscapular pain; paraesthesias; numbness and sensory changes; weakness; or abnormal deep tendon reflexes in the arm. Participants were excluded if they had either of the following: (1) myelopathy; (2) neck pain only; (3) severe diabetes or renal or liver dysfunction; (4) myopathy, neuropathy or collagen diseases; (5) no signs of root compression on cervical ultrasonographic measurements. The subjects lied at a supine position. Nerve root was scanned on cervical cross section when subject's head was tilted 45 degrees to the opposite side of the examiner with the probe gliding up and down. Note that blood vessel shoud be jundged and ruled out.

\subsection{Ultrasonographic Measurements of the Brachial Plexus}

The C5 and C6 vertebrae have both anterior tubercles and posterior tubercles. resulting in a "hill shape", or "U-shaped" signs [10]. Usually, the anterior tubercle is higher than the posterior tubercle. The anterior tubercle of the $\mathrm{C} 7$ vertebra is absent, and the outer edge of that vertebrae is flat and straight in the shape of a "slope" or "vertical thumb sign [11]. Ultrasound clearly demonstrated that the anterior tubercle of the transverse process of the C7 vertebra was small absent, even not develope, which can be used to marking of positions of the $\mathrm{C} 7$ nerve root from anatomy of vertebra [12]. The interscalene space and intervertebral foramina were useful anatomic markers in identifying the brachial plexus [13]. The positions of the cervical nerve roots were abnormal in a few subjects [14]. During the neck examination, Nerve trunk passing through traversing the intervertebral foramen, The nerve root mostly runs between the anterior diagonal line and the middle diagonal line. To ensure the measurement standard and the measurement results were accurate, the position of the intervertebral foramen was selected as the standard section in this study. They can be displayed that the osseous marks outline of the anterior and posterior tubercles of the transverse processes of the vertebrae by using high frequency ultrasound. Ultrasonic examination of the brachial plexus is effective because it can evaluate many parts of the brachial plexus and the surrounding soft tissue with high spatial resolution [15]. Ultrasonographic technicians performed ultrasonography using a real-time scanner with a $12 \mathrm{MHz}$ linear array probe within 1 weeks of the cervical MRI. All measurements were carried out bilaterally. Normally the roots, trunks and cords appear as homo-geneous, hypoechoic structures, tubular in longitudinal slices and oval in axial slices [12]. High-frequency color Doppler ultrasound exhibiting bony outline of anterior and posterior tubercles of the transverse process of the vertebra, which can choose to use 
the appropriate depth and focus.

\subsection{Statistical Analysis}

The data were analyzed with SPSS 15.0 software (SPSS, Chicago, IL, USA). Measurement data were expressed as mean $\pm \mathrm{SD}$. A comparison between left and right sides was done using paired $t$-test. One-way analysis of variance was used among different groups. Spearman correlation analysis was performed for comparison of multiple variables and measured indicators. $P<0.05$ was considered significantly significant. while the data that do not conform to normal distribution are tested by nonparametric independent samples (rank sum test), and the results are expressed by quartile. $P<0.05$ is statistically significant.

\section{Results}

Table 1 shows the imaging feasures of cervical vertebral body and intervertebral disc. The C4-C8 intervertebral discs were examined sequentially by MRI. The varying pictures of imaging characteristics of cervical vertebral body and intervertebral disc on MRI were classified into 6 types: Normal, PHC (herniation of cervical intervertebral discs), CID (cervical intervertebral discs bulging), LHC (Lateral herniation of cervical disc), RC (Root sleeve cyst), VI (Vertebral instability) respectively. The highest detection rate of PHC was C4/5, followed by C5/6, C3/4 C6/7 and C7/T1. The highest detection rate of normal intervertebral disc is C7T1 ID, which may be related to less stress on the intervertebral disc. On the contrary, the lowest detection rate of normal intervertebral disc was C5/6 ID. At C7/T1 ID, the detection rate of LHC and VI were 0 . RC was detected in C5/6ID, C6/7ID and C7/T1ID. The results are as follows: most cervical intervertebral discs presented bulging and herniation; C7/T1 intervertebral discs in most cases were normal (95.9\%), followed by C3/C4 (45.2\%), C6/C7 (36.2\%), C4/C5 (21.3\%) and C5/C6 (17.6\%). The incidence rates of anterior and posterior prominent of intervertebral discs in descending order are as follows: C4/C5 (28.1\%), C5/C6 (27.6\%), C3/C4 (21.3\%), C6/C7 (18.6\%) and C7/T1 (0.9\%), and lateral prominent of intervertebral disc was most seen in C5/C6 (10.9\%), followed by C6/C7 (5.9\%), C4/C5 (4.5\%) and $\mathrm{C} 3 / \mathrm{C} 4(3.6 \%)$, while lateral prominent was not seen in $\mathrm{C} 7 / \mathrm{T} 1$ $(0 \%)$; intervertebral disc bulging was mostly seen in $\mathrm{C} 4 / \mathrm{C} 5$ (42.1\%), followed by C5/C6 (41.6\%), C6/C7 (38\%), C3/C4 (26.2\%) and $\mathrm{C} 7 / \mathrm{T} 1$ (2.1\%); Nerve root sleeve cyst was rarely seen in $\mathrm{C} 5$ to $\mathrm{C} 7$, and its was most seen (1.9\%). Cervical intervertebral discs bulging occurred mostly at the level of C4/5 (42.1\%) and C5/6 (41.6\%). The incidence of Root sleeve cys was highest in $\mathrm{C} 7 / \mathrm{T} 1$ intervertebral discduring the head movement. Table 2 shows the diameter of cervical nerve roots in different imaging characteristics of intervertebral disc. Normal values of the diameters of cervicalr root nerves at intervertebral foramen were: $\mathrm{C} 4,2.65 \pm 0.27 \mathrm{~mm}$; C5, $3.33 \pm 0.33 \mathrm{~mm}$; C6, $3.76 \pm 0.36 \mathrm{~mm}$; C7, $4.84 \pm 0.30 \mathrm{~mm}$; C8, $3.48 \pm 0.34 \mathrm{~mm}$.

Those should be displayed althought using High-frequency color Doppler ultrasound where the brachial plexus root originates in healthy adults. The results were showed as figure. It is confirmed that the normal brachial plexus has a circular or oval hypoechoic structure on the cross section (short axial plane) (figure 1). The longitudinal section (long axis plane) imaging shows many parallel linear low-to-medium echoes with low echoes (figure 2). The image shows that it is not displayed the interal nerve bundles of the brachial plexus. It can confirm the localization of the $\mathrm{C} 7$ nerve root of noting absence of anterior tubercle at C7 level (Figure 1a). The C6 nerve root are shaped between anteriorly and posterior nodules (Figure 1b). There was no relationship between the diameter of cervical nerve roots and hypertension, blood lipid, homocysteine, smoking, alcohol consumption and body mass root index was analyzed in study. Besides, there was no correlation between cervical nerve root diameter and height, weight or age. The diameter of nerve root $\mathrm{C} 7$ was significantly larger than that of the other $(P<0.05)$, and the $\mathrm{C} 4$ diameter of nerve root was smaller than the other four nerve root $(P<0.05)$. Cervical nerve root diameter of $\mathrm{C} 4$ is significantly correlated with height, $\mathrm{p}$ value is less than 0.05 . Cervical nerve root diameter of $\mathrm{C} 4$ is significantly correlated with smoking history, and the correlation coefficient is less than 0.05 . The differences of the diameter of cervical nerve roots between vertebral body without changing group (VBWC group) and the total number of cases of cervical nerve roots (TCNR group) were not statistically significant $(P>0.05)$. There were no significant differences between the left-and right-side measurements.
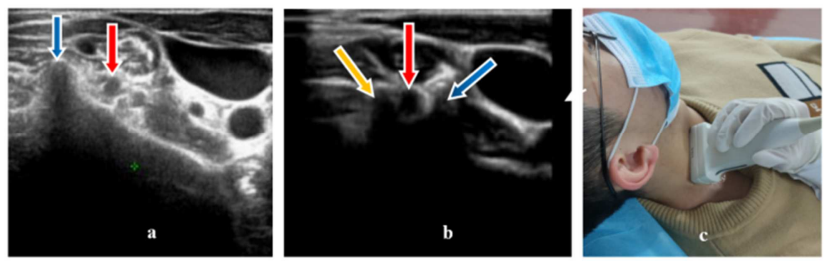

Figure 1. The ultrasonographic characteristics of nevere in short axial section.

The blue arrow indicates the posterior tubercle of $\mathrm{C} 7$ vertebra, and the red arrow indicates the $\mathrm{C} 7$ nerve roots (a). The yellow arrows indicate posterior tubercle the blue arrows indicate anterior tubercle, and the red arrows indicate C7 nerve roots (b). The probe position is shown in short axis section (c).
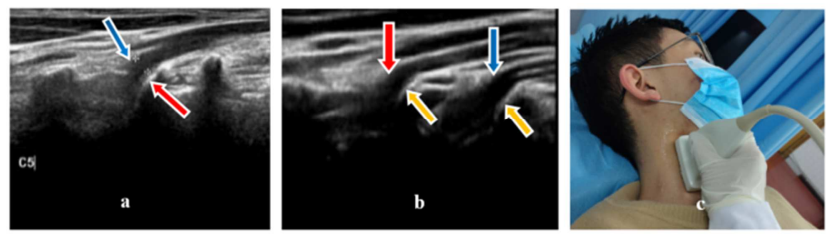

Figure 2. The ultrasonographic characteristics of nevere in long axial section.

The blue arrow indicates C5 nerve root and the red arrows indicate hyperechoic points of bone cortex in the cervical vertebrae anterior tubercle (a). The red arrow indicates the $\mathrm{C} 6$ nerve root, while the blue arrow indicates the $\mathrm{C} 7$ nerve root, the yellow arrows indicates the hyperechoic point of bone cortex in the cervical vertebrae anterior tubercle (b). The probe position is shown in long axis section (c). 


\section{Discussion}

In the last 10 years, ultrasonography has become an extremely effective examination for exploring the nerves of the upper limb [10]. Ultrasound and MRI showed a high level of agreement for the visualization of the $\mathrm{C} 6, \mathrm{C} 7$, and middle trunk segments [16]. Nerve ultrasound is becoming a standard element in the evaluation of peripheral nerve disease. Neuromuscular ultrasound (NMUS) is now used worldwide in many academic practices and gaining wider acceptance among the general neurophysiology community [17]. A high-frequency $(12+\mathrm{MHz})$ linear array transducer is primarily used in the evaluation of neuromuscular structures, Fortunately, transducers have an adjustable range of frequencies to optimize imaging [18]. The cross-sectional ultrasound imaging using a $12 \mathrm{MHz}$ linear ultrasound probe; Even if MRI remains the standard examination in the seindications and the one that offers the most promising prospects (tractography, etc.), ultrasonography with its greater clarity and its dynamic characteristics, so it is an excellent supplementary, but it has not been fully developed [10]. Recent literature has shown that a combination of high precision ultrasound imaging and electrical nerve stimulation (NS) allows for very precise location of nerve structures [19]. Ultrasound examination of the brachial plexus, although at first sight difficult, is perfectly feasible through fairly rapid practical and theoretical training.

Table 1. Imaging features of cervical vertebral body and intervertebral disc.

\begin{tabular}{llllll}
\hline \multirow{2}{*}{ Characteristics of MRI } & \multicolumn{5}{l}{ Vertebral body, intervertebral disc } \\
\cline { 2 - 6 } & C3/C4ID (F, Dr) & C4/C5ID (F, Dr) & C5/6ID (F, Dr) & C6/7ID (F, Dr) & C7/T1ID (F, Dr) \\
\hline Normal & $100,45.2 \%$ & $47,21.3 \%$ & $39,17.6 \%$ & $80,36.2 \%$ & $211,95.5 \%$ \\
PHC & $47,21.3 \%$ & $62,28.1 \%$ & $61,27.6 \%$ & $41,18.6 \%$ & $2,0.9 \%$ \\
CID & $58,26.2 \%$ & $93,42.1 \%$ & $92,41.6 \%$ & $84,38.0 \%$ & $6,2.7 \%$ \\
LHC & $8,3.6 \%$ & $10,4.5 \%$ & $24,10.9 \%$ & $13,5.9 \%$ & $0,0.0 \%$ \\
VI & $8,3.6 \%$ & $9,4.1 \%$ & $4,1.8 \%$ & $2,0.9 \%$ & $0,0.0 \%$ \\
RC & $0,0.0 \%$ & $0,0.0 \%$ & $1,0.5 \%$ & $1,0.5 \%$ & $2,0.9 \%$ \\
Total (N \%) & $221,100 \%$ & $221,100 \%$ & $221,100 \%$ & $221,100 \%$ & $221,100 \%$ \\
\hline
\end{tabular}

Note: F: frequency, Dr: Detection rate (\%), VB: Vertebral body. N: Total number of samples; posterior herniation of cervical intervertebral discs: PHC. cervical intervertebral discs bulging: CID. Lateral herniation of cervical disc: LHC. Root sleeve cyst: RC Vertebral instability: VI. C3 /C4 intervertebral disc: C3/C4ID, C4/C5 intervertebral disc: C4/C5 ID, C5/C6 intervertebral disc: C5/C6ID, C6/C7 intervertebral disc: C6/C7ID, C7/T1 intervertebral disc: C7/T1ID.

Ultrasound imaging of the brachial plexus has been compared with either magnetic resonance imaging or anatomical sections with good correlations [20]. Ultrasonogram characteristics of the brachial plexus were presented histomorphologically features. The sonographic characteristics and anatomical variation of intervertebral foramen and axillary brachial plexus were studied. Ultrasonic characteristics and anatomic variation in the intervertebral foramen and the axillary brachial plexus were investigated. During the inspection, ultrasound can be used in the assessment of the cervical nerve roots, as well as of the upper and middle trunks, although it provides limited visualization of the remaining segments. The cervical nerve roots, the upper trunk, and the middle trunk were the segments that were best visualized on ultrasound [17]. High-frequency ultrasound can clearly display the outline of the transverse processes of the vertebrae body. And the antero-posterior tubercle of the transverse process. The cervical nerve roots, upper trunk, and middle trunk were the segments that were best visualized on ultrasound [21], even under trunk. Ultrasonogram characteristics of the brachial plexus were presented histomorphologically. The nerve tract of hypo-echoes was surrounded by perineurium in nerve internal. The epineurium, with high echoes, separated nerve clusters of hypo-echoes from other structures. The epineurium should be diatinguished from the tendon. The epineurium should be diatinguished from the tendon. Ultrasound can easily show the adventitia, while high-frequency probe scanning can show the adventitia in the near field [7]. It should be noted that this appearance differs from the appearance of peripheral nerves, the fasciculated nature of which can be clearly identified with ultrasound (hypoechoic bundles embedded in more or less hyperechoic supporting connective tissue and surrounded by the hyperechoic epineurium) [10]. Some recent studies have used cervical nerve root (NR) ultrasonography for the ultrasonographic diagnosis of chronic inflammatory demyelinating polyradiculoneuropathy [22]. The examiners measured the oblique sagittal diameters of bilateral cervical nerve roots (NRs) at intervertebral foramen. Morphology of cervical nerve roots: located between the "semi-cup mouth" or "cup mouth"-shaped arc shadows, and nerve roots of C5 and C6 were the most obvious. The transverse processes of the seventh cervical vertebra and the scalene muscle space are important markers for examination. The severity of cervical disc degeneration significantly increased with age in both sexes at every level. The disc degeneration predominantly occurred at C5-C6, C4-5 and C6-C7 (in Table 1). Based on the principle of ultrasonic imaging, we should measure the maximal diameter on the short-axis section where the nerve is perpendicular to the sound beam. We should not measure the diameter on longitudinal section. Deviation may occur, because of the position of the measurement point, or as a result of difference in level or declination of the sound bea. Therefore, it should be paid attention to select of measurement points. [17]. The bony structure of the transverse process of the vertebral body passing through the intervertebral foramen of the brachial plexus nerve root clearly showed that there were strong echo bony signs on 
both sides of the hypoechoic structure. In the brachial plexus, ultrasound can be used in the assessment of the cervical nerve roots, as well as of the upper and middle trunks, although it provides limited visualization of the remaining segments. The region where the normal brachial plexus root traversed the intervertebral foramen exhibited a uniform hypo-echo. The display rate for the C4-7 nerve roots was $100 \%$, while that for $\mathrm{C} 8$ was $78 \%$. The $\mathrm{C} 7$ vertebra is a marker for identifying the location of brachial plexus nerve roots [16]. The C8 nerve root was difficult to observe in neonates [23]. The short neck meant that the examination position was insufficiently exposed, and the collarbone and lung gas limited the view. Of the 221 subjects with degeneration of cervical vertebra, $95.5 \%$ had a diagnosis of $\mathrm{C} 7 / \mathrm{T} 1$ normal intervertebral disc; the incidence of lateral herniation of cervical disc was highest among C3-T1 intervertebral discs. According to the different imaging characteristics of cervical vertebral body and intervertebral disc divided into six groups in Table 2. Results showed that the age-related risk factors (hypertension, hyperlipidemia, homocysteine, smoking, drinking and body mass index) have no relationship with the incidence of degeneration of cervical vertebra. This study showed that the diameter of cervical nerve roots did not change with age.

Table 2. The diameter of cervical nerve roots in different imaging characteristics of intervertebral disc.

\begin{tabular}{|c|c|c|c|c|c|}
\hline CNR & Groups & $\mathbf{N}$ & $\bar{x} \pm s$ & $F$ & $P$ \\
\hline \multirow[t]{4}{*}{$\mathrm{C} 4$} & Normal & 200 & $2.65 \pm 0.26$ & 1.316 & 0.269 \\
\hline & LHC & 8 & $2.81 \pm 0.39$ & & \\
\hline & PHC & 94 & $2.62 \pm 0.28$ & & \\
\hline & CID & 116 & $2.66 \pm 0.28$ & & \\
\hline \multirow[t]{4}{*}{ C5 } & Normal & 94 & $3.31 \pm 0.32$ & 1.403 & 0.241 \\
\hline & LHC & 10 & $3.20 \pm 0.33$ & & \\
\hline & PHC & 124 & $3.37 \pm 0.35$ & & \\
\hline & CID & 186 & $3.33 \pm 0.30$ & & \\
\hline \multirow[t]{4}{*}{ C6 } & Normal & 78 & $3.78 \pm 0.36$ & 3.062 & 0.028 \\
\hline & LHC & 24 & $3.59 \pm 0.27$ & & \\
\hline & PHC & 122 & $3.76 \pm 0.33$ & & \\
\hline & CID & 182 & $3.80 \pm 0.32$ & & \\
\hline \multirow[t]{4}{*}{$\mathrm{C} 7$} & Normal & 160 & $4.83 \pm 0.24$ & 0.916 & 0.433 \\
\hline & LHC & 13 & $4.87 \pm 0.19$ & & \\
\hline & PHC & 82 & $4.80 \pm 0.18$ & & \\
\hline & CID & 168 & $4.87 \pm 0.44$ & & \\
\hline \multirow[t]{3}{*}{$\mathrm{C} 8$} & Normal & 333 & $3.48 \pm 0.34$ & 0.289 & 0.749 \\
\hline & PHC & 4 & $3.60 \pm 0.16$ & & \\
\hline & CID & 11 & $3.45 \pm 0.36$ & & \\
\hline
\end{tabular}

Lateral herniation of cervical disc: LHC posterior herniation of cervical intervertebral discs: PHC. cervical intervertebral discs bulging: CID Note: N represents the diameter sample of bilateral cervical nerve

Based on the ultrasonic measurement results the $\mathrm{C} 7$ diameter of nerve root was significantly largest than the other nerve roots $(\mathrm{P}<0.05)$, and the $\mathrm{C} 4$ diameter of nerve root was smaller than the other four nerve root $(\mathrm{P}<0.05)$. The fact that the $\mathrm{C} 4$ nerve is thinner may render it more susceptible to damage during vigorous neck movement. In the experimental results, because $\mathrm{C} 4-\mathrm{C} 8$ data, height, weight and age are continuous variables, Pearson method is used to analyze their correlation. Results indicates that cervical nerve root diameter of $\mathrm{C} 4$ is significantly correlated with height, $p$ value is less than 0.05 , and the correlation coefficient is greater than 0.05 , cervical nerve root diameter of $\mathrm{C} 4$ is positively correlated with height, but not with age and weight. Cervical nerve root diameter of $\mathrm{C} 4$ is significantly correlated with smoking history, and the correlation coefficient is less than 0.05 , so cervical nerve root diameter of $\mathrm{C} 4$ is significantly negatively correlated with smoking history.

This prospective cohort study investigated the accuracy of an ultrasonographic diagnostic approach for cervical radiculopathy (NRs) detection. This goal was accomplished by measuring the CSAs of cervical NRs in 102 patients with cervical disc herniation or osteophyte compression and the comparison of these CSAs with those of 219 normal subjects
[24]. Intervertebral disc degeneration is known to occur as a result of natural aging under the influence of various environmental factors [25]. Symptomatic nerve roots are wider than asymptomatic nerve roots due to the presence of edema. Peripheral nerves have also been shown to develop edema, fibrosis, and changes distal to the affected nerve as a result of mechanical compression [26]. The shape of the intervertebral foramina approximated a funnel. The imaging examination demonstrated the most prominent characteristic features of posterior herniation of cervical intervertebral discs at $\mathrm{C} 3-\mathrm{C} 4, \mathrm{C} 4-\mathrm{C} 5$, and $\mathrm{C} 5-\mathrm{C} 6$. There were no significant differences between the left- and right-side measurements, consistent with a previous report [27]. Our study shows the same result. According to the characteristics of cervical Intervertebral disc changes the subjects were divided into Normal group, LHCgroup, PHCgroup, and CID group. There was no significant difference among the groups in Table 2. The differences of the diameter of cervical nerve roots between vertebral body without changing group (VBWC group) and the total number of cases of Cervical nerve roots (TCNR group) were not statistically significant $(\mathrm{P}>0.05)$. The display rate for the $\mathrm{C} 5-7$ nerve roots was $100 \%$, while those for $\mathrm{C} 8$ and $\mathrm{T} 1$ were $83 \%$ and $68 \%$, 
respectively [17]. The C8 nerve root display rate is $78 \%$ in our study, These findings are consistent with those in the literature in terms of the difficulty in clearly visualizing the exit passages of roots $\mathrm{C} 8$ and $\mathrm{T} 1$ [28]. The interscalene space and intervertebral foramina were useful anatomic markers in identifying the brachial plexus. Different results may be due to the selection of different populations and the small sample size. There was no difference in the diameter of cervical nerve roots between men and women $(P>0.05)$. High-frequency ultrasound is valuable in diagnosing brachial plexus closed injuries and neoplasms [13]. In our study we can accurately measure the diameter of the C5-7 nerve roots where they originate by ultrasonic. We need to measure three times, where can reduce the operating error. Ultrasonographic measurements were consistent with the ranking $\mathrm{C} 4<\mathrm{C} 5<\mathrm{C} 8<\mathrm{C} 6<\mathrm{C} 7$. The most effective way to identify the nerve root was to use the physiological characteristics of the vertebrae $[29,30]$. The C8 nerve root emerges from the C7-T1 vertebral foramen and is generally farther below the surface than the other nerve roots. Normal C5-C8 nerve roots had clear boundaries and showed different shapes at different levels, such as circular, oval, or triangular [31]. The reference values obtained in this study will facilitate the analysis abnormal nerve roots conditions, and the information on side-to-side variation and sex-specific differences should be particularly helpful.

The nerves roots of the plexus can be reliably delineated as hypoechoic structures that are tubular on longitudinal scans and rounded on transverse scans. To assess the level of individual roots, scanning first identified the $\mathrm{C} 7$ level and then moved either upward or downward on axial planes. In the present study, we have proved that the brachial plexus trunk presents zonal echo or uniform hypoecho in the longitudinal plane with regular boundary. C7 can be displayed between the scalenus anterior and the sccalenus medius of the trunk of the brachial plexus. The transverse processes of the seventh cervical vertebra, the scalene space, the subclavian artery and the deep cervical artery are important markers in an examination [16]. High echoes can be found at the edge, and the nerve exit zone was clear. Longitudinal section imaging showed many parallel linear hypo-moderate echoes. Several studies reported that age, sex, height, and weight may be correlated with the size of nerves [30]. On MRI, the degree of visualization was excellent for most of the segments. However the cervical nerve roots, upper trunk and middle trunk were the segments that were best visualized on ultrasound, other parts showed poor performance. In the comparison between ultrasound and MRI, the C6, C7, upper trunk, and middle trunk segments showed equivalent degrees of visualization, with a high level of agreement between the two methods. Ultrasound of the nerve is feasible and may be used as a non-invasive screening technique to assess nerve root integrity in brachial plexus injury. These anatomic characteristics can be used as a marker to identify the $\mathrm{C} 7$ vertebral level, where it is not consistent of that the transverse process of the $\mathrm{C} 7$ vertebra, among the anterior tubercle was absent, even not developed. The fifth to seventh cervical nerve roots (C5-7, including upper and middle trunk) were seen, whereas the eighth cervical and first thoracic nerve roots $(\mathrm{C} 8$, $\mathrm{T} 1$, including the lower trunk) were not seen in $91.7 \%(22 / 24)$ of all patients [13]. In our study these reference values may be helpful in investigating pathology of cervical spondylosisof nerve root type involving the cervical area and may be useful as an additive imaging clue for the diagnosis of cervical radiculopathy.

\section{Conclusion and Recommendation}

This study has confirmed that nerve root origin can be visible on ultrasound. Cervical disc degeneration and protrusion were frequently observed in healthy subjects. In oblique sagittal position, $\mathrm{C} 7$ was the thickest nerve root of the cervical nerve root. The $\mathrm{C} 8$ nerve root was thinner than $\mathrm{C} 7$. Disc bulging or posterior herniation had no effect on the nerve root of intervertebral foramen. $\mathrm{C} 4$ root diameter may be positively correlated with height, but not with age; $\mathrm{C} 5-8$ nerve roots traversed the intervertebral foramen and distributed outwards and downwards. Anatomical localization of the brachial plexus can facilitate observation of diagnosing traumatic brachial plexus lesions and permit accurate localization. With a high rate of visualization for the C4-C8 nerve roots, ultrasound could be used as a routine examination method for the cervical nerve roots. There were some limitations to our study. First, we enrolled 221 patients with cervical disorders, which is too small a number to offer meaningful statistical significance. We will continue to confirm the above conclusions in future study. Based on it is simple, fast, non-invasive and visual davantages that US, which will be accepted by the public.

\section{Author Contributions}

Fang Luo and Shan He provided the data, conducted experiments and provided technical or material support.

Jun Chen and Fang Luo perormed data analysis and wrote the initial draft of the manuscript.

Shan $\mathrm{Wu}$ (corresponding author) conceived and designed this study, and provided technical or material support, and revised the manuscript.

All authors have read, improved draft manuscripts and approved the final manuscript.

\section{Sources of Funding}

The study was supported by the Guiyang science and technology plan project. No. [2019] 9-1-7, National Key R\&D Program of China (NO. 2018YFC1312901). The funders had no role in study design, data collection and analysis, decision to publish, or preparation of the manuscript.

\section{Ethical Statements}

The experimental scheme was approved by the Institutional Human Study Committee of the Affiliated Hospital of 
Guizhou Medical University, China.

\section{Conflict of Interest}

The authors declare that they have no conflicts of interest concerning this article. All the authors do not have any possible conflicts of interest.

\section{Acknowledgements}

We thank the staff from the Ultrasound Room of the Department of Neurology, Affiliated Hospital of Guizhou Medical University for their help. Thanks for the support from the programme of the Guiyang science and technology plan project. No. [2019] 9-1-7, National Key R\&D Program of China (NO. 2018YFC1312901).

\section{References}

[1] Vanessa Baute, Jeffrey A Strakowski, Jon W Reynolds, et al. Neuromuscular ultrasound of the brachial plexus: A standardized approach. Muscle Nerve. 2018; 58: 618-24.

[2] J-L Christophe, F Berthier, A Boillot, et al. Assessment of topographic brachial plexus nerves variations at the axilla using ultrasonography. Br J Anaesth. 2009; 103: 606-12.

[3] Daniel Boczar, Antonio J Forte, Jeremie D Oliver, et al. Intraoperative evaluation of cervical nerve root avulsion using ultra-high-frequency ultrasound system. Case Reports Plast Surg Hand Surg. 2019; 6: 43-46.

[4] O Kenechi Nwawka. Ultrasound Imaging of the Brachial Plexus and Nerves About the Neck. Ultrasound Q. 2019; 35: 110-19.

[5] Khalid M Abbed, Jean-Valéry C E Coumans. Cervical radiculopathy: pathophysiology, presentation, and clinical evaluation. Neurosurgery. 2007; 60: S28-34.

[6] James F Griffith, Radhesh Krishna Lalam. Top-Ten Tips for Imaging the Brachial Plexus with Ultrasound and MRI. Semin Musculoskelet Radiol. 2019; 23: 405-18.

[7] Zhengyi Li, Xun Xia, Xiaoming Rong, et al. Structure of the brachial plexus root and adjacent regions displayed by ultrasound imaging. Neural Regen Res. 2012; 7: 2044-50.

[8] Royse CE, Sha S, Soeding PF, et al. Anatomical study of the branchial plexus using surface ultrasound. Anaesth Intensive Care. 2006; 34: 203-10.

[9] Rune Lau Jakobsen, Anders Fuglsang-Frederiksen, Michel Bach Hellfritzsch, et al. A prospective study of high resolution ultrasound in brachial plexopathies-Correlation with electrophysiological measurements. Clin Neurophysiol. 2019; 130: $1144-50$.

[10] Lapegue F, Faruch-Bilfeld M, Demondion X, et al. Ultrasonography of the brachial plexus, normal appearance and practical applications. Diagn Intervent Imaging. 2014; 95: 259-75.

[11] Griffith JF. Ultrasound of the brachial plexus. Semin Musculoskelet Radiol. 2018; 22: 323-33.

[12] Martinoli C, Bianchi S, Santacroce E, et al. Brachial plexus sonography: a technique for assessing the root level. AJR Am J
Roentgenol. 2002; 179: 699-2.

[13] Minjuan Zheng, Yongsheng Zhu, Xiaodong Zhou, et al. Diagnosis of closed injury and neoplasm of the brachial plexus by ultrasonography. J Clin Ultrasound. 2014; 42: 417-22.

[14] Wenying Wang, and Qiao Wang. Sonographic measurements of normal C5-C8 nerve roots in children. Muscle Nerve. 2020; 61: 649-53.

[15] James F Griffith. Ultrasound of the Brachial Plexus Semin. Musculoskelet Radiol. 2018; 22: 323-33.

[16] Kubiena H, Hormann M, Michlits W, et al. Intraoperative imaging of the brachial plexus by high-resolution ultrasound. J Reconstr Microsurg. 2005; 21: 429-33.

[17] Caldana WCI, Kodaira SK, Cavalcanti CFA, et al. Value of ultrasound in the anatomical evaluation of the brachial plexus: correlation with magnetic resonance imaging. Radiol Bras. 2018; 51: 358-65.

[18] Gonzalez NL, Hobson-Webb LD. Neuromuscular ultrasound in clinical practice: A review. Clin Neurophysiol Pract. 2019; 4: $148-63$.

[19] Perlas A, Chan VW, Simons M. Brachial plexus examination and localization using ultrasound and electrical stimulation: a volunteer study. Anesthesiology. 2003; 99: 429-35.

[20] Demondion X, Herbinet P, Boutry N, et al. Sonographic mapping of the normal brachial plexus. Am J Neuroradiol. 2003; 24: 1303-09.

[21] Zaidman CM, Al-Lozi M, Pestronk A. Peripheral nerve size in normals and patients with polyneuropathy: an ultrasound study. Muscle Nerve. 2009; 40: 960-66.

[22] Smith EC, Xixis KI, Grant GA, et al. Assessment of obstetric plexus injury with preoperative ultrasound. Muscle Nerve. 2016; 53: 946-50.

[23] Padua L, Granata G, Sabatelli M, et al. Heterogeneity of root and nerve ultrasound pattern in CIDP patients. Clin Neurophysiol. 2014; 125: 160-65.

[24] Mikinobu Takeuchi, Norimitsu Wakao, Atsuhiko Hirasawa, et al. Ultrasonography has a diagnostic value in the assessment of cervical radiculopathy: A prospective pilot study. Eur Radiol. 2017; 27: 3467-73.

[25] Gore DR. Roentgengraphic findings in the cervical spine in asymptomatic persons: a ten-year follow-up. Spine (Phila Pa 1976). 2001; 26: 2463-66.

[26] Burcu Metin Ökmen, Korgün Ökmen, Lale Altan. Investigation of the Effect of Cervical Radiculopathy on Peripheral Nerves of the Upper Extremity with High-Resolution Ultrasonography. Spine (Phila Pa 1976). 2018; 15; 43: E798-803.

[27] Won SJ, Kim BJ, Park KS, et al. Measurement of cross-sectional area of cervical roots and brachial plexus trunks. Muscle Nerve. 2012; 46: 711-16.

[28] Chen Y, Kumar N, Lim JW, et al. High-resolution sonography detects extraforaminal nerve pathology in patients initially diagnosed with cervical disc disease: a case series. J Clin Ultrasound. 2013; 41: 46-54.

[29] Wenying Wang, Qiao Wang. Sonographic measurements of normal C5-C8 nerve roots in children. Muscle Nerve. 2020; 61: 649-53. 
[30] Franco CD, Williams JM. Ultrasound-guided interscalene block reevaluation of the "stoplight" sign and clinical implications. Reg Anesth Pain Med. 2016; 41: 452-59.
[31] Vanderhave KL, Brighton B, Casey V, et al. Applications of musculoskeletal ultrasonography in pediatric patients. J Am Acad Orthop Surg. 2014; 22: 691-98. 